\title{
Journal of Bone and Mineral Metabolism Best Paper Award 2010
}

(C) The Japanese Society for Bone and Mineral Research and Springer 2011

The Journal of Bone and Mineral Metabolism Best Paper Award was established in 2008. Candidates for the award must be members of the Japanese Society of Bone and Mineral Research, and the winner is honored at the Society's Annual Meeting.

We are pleased to announce that the following article has received JBMM Best Paper Award.

"Oxygen tension is an important mediator of the transformation of osteoblasts to osteocytes"

by

Makoto Hirao, Jun Hashimoto, Naomi Yamasaki, Wataru Ando, Hideki Tsuboi,

Akira Myoui, Hideki Yoshikawa

J Bone Miner Metab (2007) 25:266-276

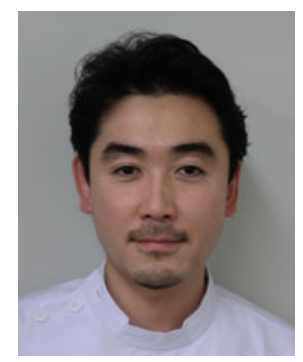

Dr. Makoto Hirao

\begin{abstract}
Osteocytes are derived from osteoblasts, but reside in the mineralized bone matrix under hypoxic conditions. Osteocyte-like cells show higher expression of ORP150, which is induced by hypoxia, than osteoblast-like cells. Accordingly, we hypothesized that the oxygen tension may regulate the transformation of osteoblasts to osteocytes. MC3T3-E1 cells and calvariae from 4-day-old mice were cultured under normoxic $\left(20 \% \mathrm{O}_{2}\right)$ or hypoxic $\left(5 \% \mathrm{O}_{2}\right)$ conditions. To investigate osteoblastic differentiation and transformation to osteocytes, alizarin red staining was done and the expression of various factors was assessed. Hypoxic culture promoted the increased synthesis of mineralized matrix by MC3T3-E1 cells. Alkaline phosphatase activity was initially increased during hypoxic culture, but decreased during osteogenesis. Osteocalcin production was also increased by hypoxic culture, but decreased after mineralization. Furthermore, expression of Dmp1, Mepe, Fgf23, and Cx43, which are osteocyte-specific or osteocyte predominant proteins, by MC3T3-E1 cells was greater under hypoxic than under normoxic conditions. In mouse calvarial cultures, the number of cells in the bone matrix and cells expressing Dmp1 and Mepe were increased by hypoxia. In MC3T3-E1 cell cultures, ORP150 expression was only detected in the mineralized nodules under normoxic conditions, while its expression was diffuse under hypoxic conditions, suggesting that the nodules were hypoxic zones even in normoxic cultures. These findings suggest that a low oxygen tension promotes osteoblastic differentiation and subsequent transformation to osteocytes.
\end{abstract}

We offer our sincere congratulations on behalf of the Journal of Bone and Mineral Metabolism, with best wishes for further development of the author's research.

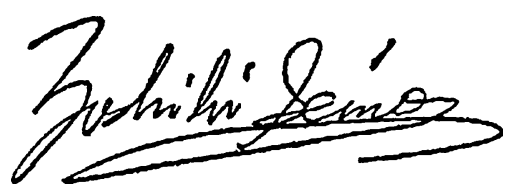

Yoshiki Seino

Editor-in-Chief

Journal of Bone and Mineral Metabolism 